Cite this: Org. Biomol. Chem., 2013, 11, 5877

Received 28th May 2013,

Accepted 12th July 2013

DOI: $10.1039 / c 3 o b 41112 e$

www.rsc.org/obc

\title{
Unprecedented regiochemical control in the formation of aryl[1,2-a]imidazopyridines from alkynyliodonium salts: mechanistic insights $\dagger$
}

\author{
Luke I. Dixon, ${ }^{a}$ Michael A. Carroll, ${ }^{\text {a }}$ Thomas J. Gregson, $\neq^{\mathrm{b}}$ George J. Ellames, $\neq^{\mathrm{b}}$ \\ Ross W. Harrington ${ }^{a}$ and William Clegg ${ }^{a}$
}

\begin{abstract}
Aryl(alkynyl)iodonium salts have been demonstrated to be valuable precursors to a diverse range of heteroaromatic ring systems including aryl[1,2-a]imidazopyridines. Successful application, using the recently described aryl(alkynyl)iodonium trifluoroacetate salts, is described, highlighting for the first time that the regioselectivity of this process is both counter-ion and concentration dependent. Studies with a carbon13 labelled substrate established that the reactions of alkynyliodonium salts are highly complex and that multiple mechanistic processes appear to be underway simultaneously.
\end{abstract}

\section{Introduction}

Alkynyliodonium salts, first discovered in 1965 by Beringer and Galton, ${ }^{1}$ are a highly versatile class of compounds and have found widespread application in both organic and inorganic syntheses and as such have been the subject of numerous reviews. ${ }^{2-10}$

As highly electron-deficient acetylenic species, alkynyliodonium salts are reactive partners in cycloaddition reactions ${ }^{3}$ including 1,3-dipolar cycloadditions ${ }^{11-14}$ and Diels-Alder chemistry. ${ }^{15-17}$ The hypernucleofuge nature of the iodoarene in alkynyliodonium salts also makes them excellent sources of carbenes, ${ }^{10}$ providing access to a wealth of cyclic species such as cyclopentenes ${ }^{18,19}$ and pyrroles; ${ }^{20}$ recently highly synthetically challenging cyanocarbenes have also been generated. ${ }^{21,22}$

In the same fashion, indoles, furopyridines, indenes, imidazopyridines, imidazopyrimidines, furotropones, furonaphthoquinones, thiazoles, selenazoles and benzofurans (Scheme 1) may also be formed. ${ }^{2,4}$ Cumulatively, these ring systems account for a wide range of known pharmacophores, yet the potential for alkynyliodonium salts in the preparation of heterocycles remains to be exploited.

\footnotetext{
${ }^{a}$ School of Chemistry, Newcastle University, Newcastle upon Tyne, NE1 7RU, UK. E-mail: m.a.carroll@ncl.ac.uk; Fax: +44 (0)191222 6929

${ }^{b}$ Department of Isotope Chemistry and Metabolite Synthesis, sanofi-aventis, Alnwick, Northumberland NE66 $2 \mathrm{JH}, \mathrm{UK}$

†Electronic supplementary information (ESI) available: Full experimental details and spectra, tables of X-ray crystallographic data and results. CCDC 907271-907274. For ESI and crystallographic data in CIF or other electronic format see DOI: 10.1039/c3ob41112e

$\ddagger$ Current address: Department of Isotope Chemistry, Covance Laboratories, Alnwick, Northumberland, NE66 2JH, UK.
}

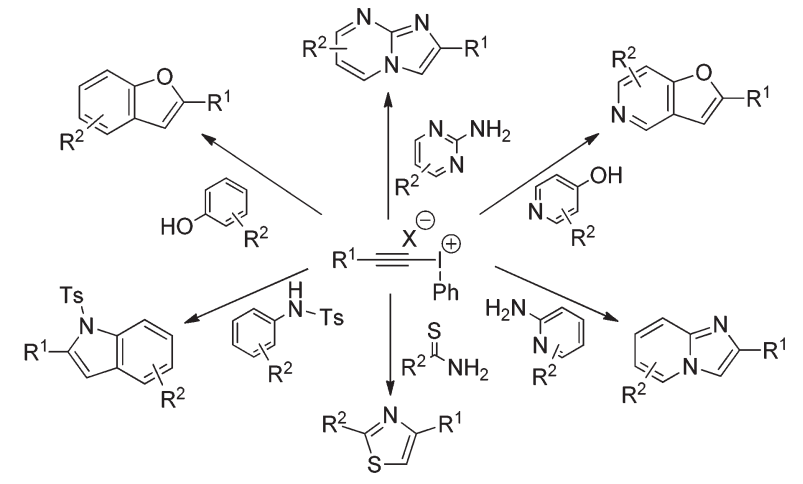

Scheme 1 Heteroaromatics from alkynyliodonium salts. ${ }^{2,4}$

Commonly used anions in alkynyliodonium salts include mesylates, ${ }^{23}$ tosylates, ${ }^{24,25}$ triflates $^{26,27}$ and tetrafluoroborates $^{28,29}$ due to their low nucleophilicity, though in many of these cases addition of the anion to the $\beta$-acetylenic position was still observed. ${ }^{30}$ As such the development of alkynyliodonium salts with an intramolecular anion has been an area of continuing interest as a means of restraining the addition reaction. ${ }^{30-36}$ In contrast, alkynyliodonium trifluoroacetates (TFA) have received little attention, ${ }^{3,37}$ though it was recently shown by us that they are not only readily prepared ${ }^{38}$ but also available on a large scale $(>0.5 \mathrm{~mol}) .{ }^{39}$

Despite the wealth of alkynyliodonium salts reported to date, very little is known about the effects of the counter-ion used or their solution state behaviour. A novel comparison is presented herein between the TFA salts and some of the more common alkynyliodonium salts, demonstrating for the first time that the anion used imparts a profound effect on the 


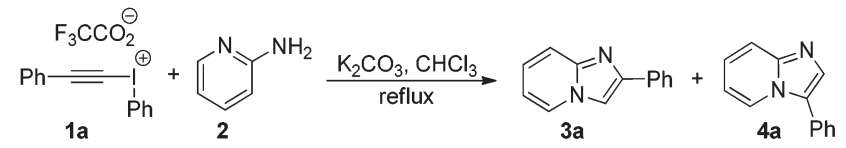

Scheme 2 Synthesis of imidazo[1,2-a]pyridines from $\mathbf{1 a}$.

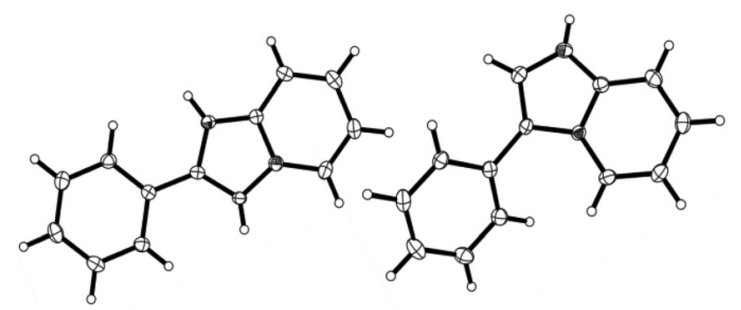

Fig. 1 Structures of the cations of $\mathbf{3 a} \cdot \mathbf{H C l} \cdot \mathrm{H}_{2} \mathrm{O}$ (left) and $\mathbf{4 a} \cdot \mathrm{HCl} \cdot 2 \mathrm{H}_{2} \mathrm{O}$ (right), with $40 \%$ probability displacement ellipsoids; $\mathrm{N}$ atoms are shown with shading.

regioselectivity of arylimidazo[1,2-a]pyridine formation; it was also found that the outcome of the reaction could be manipulated through substrate concentration. This study highlights a dynamic solution state for alkynyliodonium salt derivatives and that, through control of experimental conditions, access to a plethora of substituted heteroaromatics may be achieved, with alternative regioisomers possible from the same starting material.

In 2004 Liu and co-workers reported the synthesis of a range of 2-arylimidazo[1,2- $a]$ pyridines $(c f$. 3a) from the reaction of alkynyliodonium tosylates and 2-aminopyridine. ${ }^{40}$ Surprisingly, the analogous reaction of 1a afforded both 2- and 3-substituted imidazo[1,2- $a$ ]pyridines in roughly equal amounts (Scheme 2), as confirmed by X-ray crystallography (ESI $\dagger$ and Fig. 1).

This intriguing production of two regioisomers led us to repeat the procedure reported by Liu and co-workers ${ }^{40}$ using the tosylate, $\mathbf{5}$, to confirm that just one regioisomer had indeed been produced. In our hands, the experiment showed that the major product of the reaction was in fact $\mathbf{4 a}$ and that, although this was dominant, trace amounts of $3 \mathbf{a}$ were also present $(2 \%, 3 \mathbf{a}: 44 \%, 4 \mathbf{a})$; comparison of the ${ }^{1} \mathrm{H}-\mathrm{NMR}$ data with other reports supports this regiochemical assignment. ${ }^{41-44}$ Having optimized the reaction shown in Scheme 2 for a range of solvents and bases (see ESI $\dagger$ ), fluorobenzene (PhF) (to minimise intermolecular insertion) and $\mathrm{K}_{2} \mathrm{CO}_{3}$ were chosen respectively, and at room temperature to minimize decomposition of the alkynyliodonium salts.

Heterogeneous bases and aprotic, ${ }^{45}$ non-coordinating solvents gave the best results. Despite slightly lower yields, PhF was chosen as it resulted in a 'cleaner' reaction facilitating isolation of the products. Comparison with other alkynyliodonium salts showed that 3a was undetectable using the triflate (6), whereas the cyclic iodane, 7 (see $\mathrm{ESI}^{\dagger}$ ), produced a ratio of products between that observed for the trifluoroacetate and tosylate salts (Table 1). Only the phenyliodonium derivatives were investigated since variation of the second aromatic ring was previously found to have no effect in the preparation of 2-arylfuro[3,2-c]pyridines. ${ }^{38}$
Table 1 Counter-ion dependence for the reaction of phenyl(phenyl-ethynyl)iodonium salts and $\mathbf{2}$

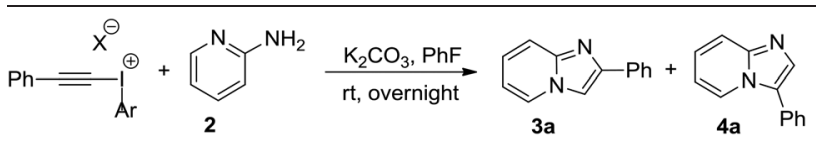

\begin{tabular}{lcl}
\hline Ar, X & Ratio 3a: 4a & Total yield $^{a}(\%)$ \\
\hline Ph, TFA (1a) & $73: 27$ & $49^{b}$ \\
Ph, OTs (5) & $7: 93$ & 62 \\
Ph, OTf (6) & $0: 100$ & 58 \\
$\mathrm{C}_{6} \mathrm{H}_{4}-2-\mathrm{CO}_{2}{ }^{-},-(7)^{c}$ & $37: 63$ & 54
\end{tabular}

${ }^{a}$ Isolated yields. ${ }^{b}$ Mean of 3 syntheses. ${ }^{c}$ Temp. raised to reflux after $14 \mathrm{~h}$.

Such a dependence on the anion used has never been reported previously in the formation of heteroaromatics from alkynyliodonium salts, though the literature suggests that the conversion of alkynyliodonium salts to alkenyliodonium salts appears to demonstrate stereoselectivity for the $E$ - or $Z$-isomer depending on the counter-ion used..$^{45-55}$ The influence of solvent on ion pair separation, and hence choice of counterion, has also been shown to affect the reactions of diaryliodonium salts. ${ }^{56}$

In addition to the counter-ion dependence of the reaction it was found that the concentration of the reactants also exerted an unexpected degree of control over the product distribution (Table 2 and Fig. 2).

It should be noted that 1a was soluble in DCM and $\mathrm{CHCl}_{3}$ at all the concentrations tested, though not in PhF; however, in all three solvents, rapid dissolution of 1a was observed on addition of 2 . Under the reaction conditions outlined in Scheme 1 the $\mathrm{K}_{2} \mathrm{CO}_{3}$ remained as a solid throughout. This suggests that the key reactive species is generated in situ as the same concentration dependence was observed for all three solvents (see $\mathrm{ESI}^{\dagger}$ ).

Although some increase in yield was observed at higher dilutions, far more noticeable was the concentration-dependent regioselectivity favouring the 2 -substituted regioisomer, 3a. To confirm this unexpected dependence, reactions were conducted using five different iodonium salt concentrations (Table 2 and Fig. 2). This 'tuning' of the reaction conditions has potential value, for example in drug discovery, where both regioisomers are accessible from a single set of reagents.

Table 2 Concentration dependence for the reaction of $\mathbf{1 a}$ and $\mathbf{2}$

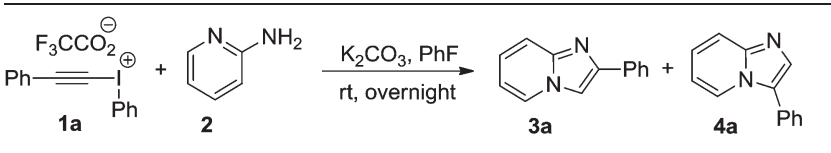

\begin{tabular}{lll}
\hline$[\mathbf{1 a}]\left(\mathrm{mol} \mathrm{L}^{-1}\right)$ & Ratio 3a : 4a & Total yield $^{a, b, c}(\%)$ \\
\hline 0.01 & $90: 10$ & 49 \\
0.02 & $73: 27$ & 49 \\
0.11 & $39: 61$ & 50 \\
0.50 & $26: 74$ & 34 \\
1.00 & $17: 83$ & 38
\end{tabular}

${ }^{a}$ Isolated yields. ${ }^{b}$ Mean of 3 syntheses. ${ }^{c} 5$ mmol scale. 


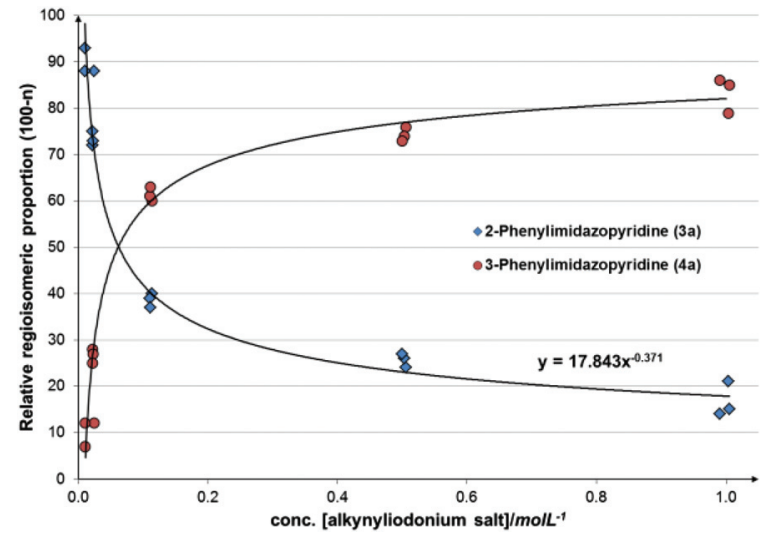

Fig. 2 Concentration dependence for the reaction of $\mathbf{1} \mathbf{a}$ and $\mathbf{2}$.

To establish whether the observed concentration dependence was restricted to the production of phenylimidazo[1,2-a]pyridines ( $\mathbf{3 a}$ and $\mathbf{4 a}$ ) several alternative alkynyliodonium salts were also studied (1b-1d) and it was pleasing to note that a similar trend was found (Table 3).

The mechanism of imidazopyridine formation presented by $\mathrm{Liu}^{40}$ follows that previously reported by Wipf ${ }^{57}$ (and later $\mathrm{Togo}^{30}$ ) for the formation of thiazoles. An alternative route to the observed product has also been proposed by Ochiai. ${ }^{58}$ All of these options invoke a monomeric form of the aryl(alkynyl)iodonium salt as the starting species even though kinetic and spectroscopic evidence for other hypervalent iodine compounds has been reported that indicates the presence, in solution, not only of associated counter-ions, but also of higher-order structures (dimers, oligomers etc.). ${ }^{59-61}$ Such species have also been shown to be highly concentration-dependent ${ }^{60,61}$ and therefore contributions from these structures cannot be ruled out.

In addition these proposals rapidly result in loss of the counter-ion. However, to retain the influence of this component, and taking into account these prior mechanistic studies, we propose that intermediates such as $\mathbf{9}$ and $\mathbf{1 4}$ (Fig. 3) and $\mathbf{8}$ and 13 (Fig. 4) should also be considered in the mechanistic rationale since both the amino- and pyridinyl-nitrogen atoms of 2aminopyridine are viable nucleophiles ${ }^{62}$ (Scheme 3: there may also be influence of the counter-ion in the subsequent steps due to the charged nature of the proposed intermediates).

Table $\mathbf{3}$ Concentration dependence for the reaction of $\mathbf{1 b} \mathbf{b} \mathbf{1 d}$ and $\mathbf{2}$

\begin{tabular}{llll}
\hline 1b-1d & & & \\
\hline Ar & {$[\mathbf{1}]\left(\mathrm{mol} \mathrm{L}^{-1}\right)$} & Ratio $3: \mathbf{4}^{a}$ & Total yield $^{b}(\%)$ \\
\hline 4-Methylphenyl (1b) & 0.02 & $74: 26$ & 66 \\
4-Methylphenyl (1b) & 0.10 & $44: 54$ & 63 \\
3'-Thienyl (1c) & 0.02 & $84: 16$ & 60 \\
3'-Thienyl (1c) & 0.14 & $57: 43$ & 58 \\
4-Bromophenyl (1d) & 0.02 & $81: 19$ & 78 \\
4-Bromophenyl (1d) & 0.14 & $53: 47$ & 55
\end{tabular}

${ }^{a} 2.5$ mmol scale. ${ }^{b}$ Isolated yields.
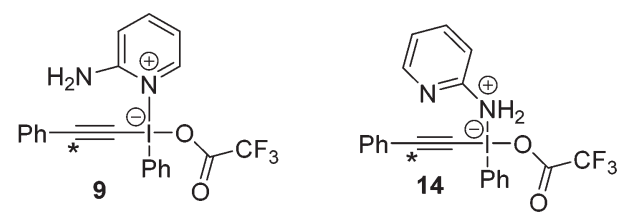

Fig. 3 Proposed [10-I-4] intermediates, 14 and 18

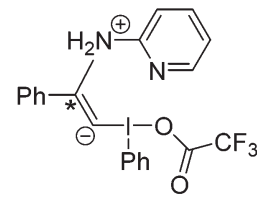

8

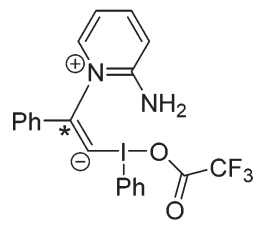

13
Fig. 4 Potential intermediates of Michael addition.

Further complexity is introduced following alkylidene carbene formation (the carbene can either cyclize directly or undergo 1,2-migration prior to cyclization), resulting in the formation of 3a or 4a via several different pathways.

As the acetylenic products of 1,2-migration have been reported to be highly reactive, ${ }^{58,63}$ especially within a basic environment, they may prove difficult to observe and as such we prepared the isotopically labelled $\left[7^{\prime}-{ }^{13} \mathbf{C}\right]-\mathbf{1 a}$ to investigate the process.

This preliminary ${ }^{13} \mathrm{C}$-labelling study generated $\left[2-{ }^{13} \mathrm{C}\right]-3 \mathbf{a}$ and $\left[3^{-13} \mathbf{C}\right]-\mathbf{4 a}$ as expected (Scheme 3); however, the isotopomer $\left[33^{-13} \mathbf{C}\right]-3 \mathbf{a}$ was also present, highlighting that a competitive 1,2-migration was occurring (Scheme 4) and suggesting that at least three reaction pathways are in operation.

\section{Conclusions}

In summary, we have presented the first example of regiochemical control in the synthesis of heteroaromatics from alkynyliodonium salts. A protocol based on the counter-ion and concentration dependence of the process has been identified for the selective formation of 2-arylimidazo[1,2-a]pyridines and 3-arylimidazo[1,2-a]pyridines. In addition, initial studies using carbon-13 labelled substrates have demonstrated that, even though well studied, the reactions of alkynyliodonium salts are highly complex and that multiple mechanistic processes appear to be underway simultaneously.

This new-found understanding is being applied to the preparation of a diverse range of heterocyclic ring systems which are of interest to our drug discovery programmes. Further work to resolve and differentiate the many mechanistic options is on-going.

\section{Experimental}

Reactions requiring anhydrous conditions were performed using oven- or flame-dried glassware and conducted under a 


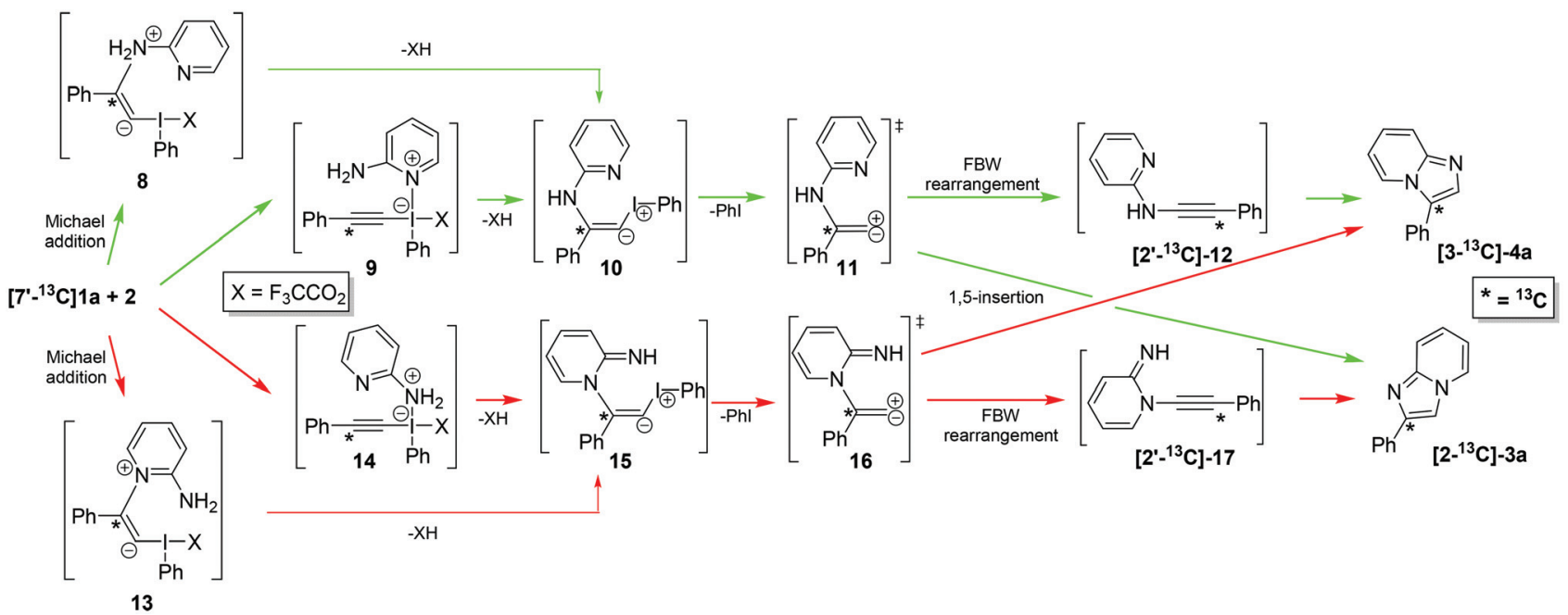

Scheme 3 Distribution of products from the reaction of $\left[7^{\prime}-{ }^{13} \mathrm{C}\right]-1$.

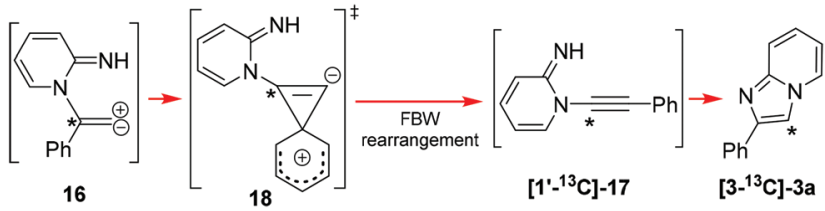

Scheme 4 Mechanism of isotopomer formation; $\left[3-{ }^{13} \mathrm{C}\right]-3 \mathrm{a}$

positive pressure of nitrogen. Anhydrous solvents were prepared thus: DCM and MeCN were refluxed over $\mathrm{CaH}_{2}$; THF, ether and hexane were refluxed over sodium/benzophenone; toluene was refluxed over sodium; and dibromomethane, chloroform, 1,4-dioxane and fluorobenzene were stored over $3 \AA$ molecular sieves. Infrared spectra were recorded on a Varian Scimitar Series 800 FT-IR with internal calibration. ${ }^{1} \mathrm{H}$ and ${ }^{13} \mathrm{C}$-NMR spectra were recorded on a Bruker Advance $300 \mathrm{MHz}$ spectrometer, a Jeol ECS $400 \mathrm{MHz}$ spectrometer or a Jeol Lamda $500 \mathrm{MHz}$ spectrometer with residual tetramethylsilane solvent as the reference for ${ }^{1} \mathrm{H}$ and ${ }^{13} \mathrm{C}$. All coupling constants are given in $\mathrm{Hz}$. Elemental analyses were carried out at London Metropolitan University. Mass spectrometry was recorded at the EPSRC Mass Spectrometry Service, Swansea or on a Waters LCT Premier (TOF-MS) operating in ' $\mathrm{W}$ ' mode. Melting points were recorded on a Gallenkamp MF-370 melting point apparatus and are uncorrected. Automated flash chromatography was performed using a Varian IntelliFlash 971-FP discovery scale flash purification system. The terms 'ether' and 'petrol' refer to diethyl ether and the fractions boiling between 40 and $60{ }^{\circ} \mathrm{C}$ (unless otherwise specified) respectively. X-ray crystallographic data were measured on an Agilent Technologies Gemini A Ultra diffractometer at $150 \mathrm{~K}$, using Mo or CuK $\alpha$ radiation; full details are in the ESI $\dagger$ and deposited with CCDC.

CAUTION: Some hypervalent iodanes are potentially explosive and should be handled taking appropriate precautions. ${ }^{64-67}$

\section{2-Phenylimidazo[1,2-a]pyridine (3a) $)^{40,68,69}$}

$\mathrm{K}_{2} \mathrm{CO}_{3}(1.05 \mathrm{~g}, 7.62 \mathrm{mmol})$ and $2(0.31 \mathrm{~g}, 3.27 \mathrm{mmol})$ were stirred together in dry $\mathrm{PhF}(250 \mathrm{~mL})$ for $45 \mathrm{~min}$ before the addition of $1 \mathrm{a}(1.05 \mathrm{~g}, 2.51 \mathrm{mmol})$ by powder funnel. The solution was then stirred in darkness, at RT, under nitrogen overnight before being washed with water $(300 \mathrm{~mL})$ and extracted into DCM $(2 \times 75 \mathrm{~mL})$. The organic fractions were dried $\left(\mathrm{MgSO}_{4}\right)$ and concentrated in vacuo to give a brown oil. The crude product was purified by column chromatography $\left(\mathrm{SiO}_{2}\right.$, Grace Resolve ${ }^{\mathrm{TM}} 80 \mathrm{~g}$ cartridge; $\S$ sample loaded in DCM, 1:0 hexane-ether for $5 \mathrm{~min}$ then increasing to $3: 7$ over $120 \mathrm{~min}$ and holding at this solvent mixture until elution was complete) to give the product as a white crystalline solid (0.24 g, $1.22 \mathrm{mmol}, 49 \%)$. Mp 132-133 ${ }^{\circ} \mathrm{C}$ (from DCM-petrol) (lit., ${ }^{70} 136-137{ }^{\circ} \mathrm{C}$ from cyclohexane); $R_{\mathrm{f}} 0.55$ (4:1 etherpetrol); Found: C, 80.4; H, 5.3; N, 14.4. Calc. for $\mathrm{C}_{13} \mathrm{H}_{10} \mathrm{~N}_{2}$ : C, 80.4; H, 5.2; N, 14.4\%; IR $\nu_{\max } / \mathrm{cm}^{-1}$ (neat) 3130, 1632, 1502, 1475, 1447, 1369, 1353, 1304, 1273, 1246, 1203, 1145, 1077, $1027 ; \delta_{\mathrm{H}}\left(300 \mathrm{MHz}, \mathrm{CDCl}_{3} ; \mathrm{Me}_{4} \mathrm{Si}\right) 8.10(1 \mathrm{H}, \mathrm{d}, \mathrm{H} 5 \mathrm{~J} 6.9), 7.97$ (2H, d, H2'/H6' J 7.2), 7.85 (1H, s, H3), 7.65 (1H, d, H8 J 9.0), $7.45\left(2 \mathrm{H}, \mathrm{t}_{\mathrm{app} .}, \mathrm{H}^{\prime} / \mathrm{H}^{\prime} J\right.$ 7.5), $7.34\left(1 \mathrm{H}, \mathrm{t}, \mathrm{H} 4^{\prime} J\right.$ 7.5), $7.17(1 \mathrm{H}$, $t_{\text {app. }}, \mathrm{H} 7 J$ 6.9), $6.77\left(1 \mathrm{H}, \mathrm{t}_{\mathrm{app} .}, \mathrm{H} 6 J 6.0\right) ; \delta_{\mathrm{C}}\left(75 \mathrm{MHz}, \mathrm{CDCl}_{3}\right.$; $\left.\mathrm{Me}_{4} \mathrm{Si}\right) 146.41$ (C2), 146.09 (C9), 134.27 (C1'), 128.99 (C3'/C5'), 128.27 (C4'), $126.55 \mathrm{C}^{\prime} / \mathrm{C6}^{\prime}$ ), 125.86 (C5), 124.79 (C7), 118.00 (C8), 112.66 (C6), 108.38 (C3); $m / z$ (CI) 195 ([M + H] $\left.]^{+}, 100 \%\right)$, 95 (3), 80 (2), 52 (4). Found: $[\mathrm{M}+\mathrm{H}]^{+}, 195.0917 . \mathrm{C}_{13} \mathrm{H}_{11} \mathrm{~N}_{2}$ requires 195.0917.

\section{3-Phenylimidazo[1,2-a]pyridine $(4 a)^{43,71}$}

Using $\mathrm{K}_{2} \mathrm{CO}_{3}(2.15 \mathrm{~g}, 15.55 \mathrm{mmol}), 2$-aminopyridine $(0.61 \mathrm{~g}$, $6.50 \mathrm{mmol}), \mathrm{PhF}(5 \mathrm{~mL})$ and $1 \mathrm{a}(2.07 \mathrm{~g}, 4.95 \mathrm{mmol})$. White crystalline solid (0.36 g, $1.84 \mathrm{mmol}, 37 \%)$. Mp $95-97{ }^{\circ} \mathrm{C}$ (from

$\S$ Although several column packings were evaluated, e.g. reverse phase silica, alumina (neutral, basic and acidic), with a range of solvents and additives, the Grace cartridges were found to provide satisfactory purification. 
$\mathrm{MeOH}-\mathrm{H}_{2} \mathrm{O}$ ) (lit., ${ }^{71} 97-98{ }^{\circ} \mathrm{C}$ from petroleum ether); $R_{\mathrm{f}} 0.13$ (4:1 ether-petrol); Found: C, 80.3; H, 5.1; N, 14.3. Calc. for $\mathrm{C}_{13} \mathrm{H}_{10} \mathrm{~N}_{2}$ : C, 80.4; H, 5.2; N, 14.4\%; IR $\nu_{\max } / \mathrm{cm}^{-1}$ (neat) 1634, 1603, 1540, 1499, 1480, 1450, 1442, 1352, 1296, 1272, 1262, $1175,1148,1134,1074,1009 ; \delta_{\mathrm{H}}\left(400 \mathrm{MHz}, \mathrm{d}_{6}-\mathrm{DMSO} ; \mathrm{Me}_{4} \mathrm{Si}\right)$ 8.45 (1H, d, H5 J 6.9), 7.72 (1H, s, H2), 7.61 (1H, d, H8 $J$ 8.7), 7.57 (2H, d, H2'/H6' J 7.3), 7.46 (2H, tapp., H3'/H5' J 7.8), 7.34 (1H, t, H4' J 7.4), 7.21 (1H, tapp. H7 J 7.8), 6.86 (1H, tapp., H6 J 6.6); $\delta_{\mathrm{C}}\left(100 \mathrm{MHz}, \mathrm{d}_{6}\right.$-DMSO; $\left.\mathrm{Me}_{4} \mathrm{Si}\right) 146.06$ (C9), 133.07 (C2), 129.74 (C3'/C5'), 129.42 (C1'), 128.37 (C4'), 127.97 (C2'/C6'), 125.58 (C3), 125.00 (C7), 124.45 (C5), 118.09 (C8), 113.30 (C6); $m / z$ (ESI) $195\left([\mathrm{M}+\mathrm{H}]^{+}, 100 \%\right)$. Found: $[\mathrm{M}+\mathrm{H}]^{+}, 195.0905$. $\mathrm{C}_{13} \mathrm{H}_{11} \mathrm{~N}_{2}$ requires 195.0922 .

\section{2-(4'-Methylphenyl)imidazo[1,2-a]pyridine $(3 b)^{69,72}$}

Using $\mathrm{K}_{2} \mathrm{CO}_{3}(1.09 \mathrm{~g}, 7.89 \mathrm{mmol}), 2$ (0.32 g, $\left.3.39 \mathrm{mmol}\right), \mathrm{PhF}$ $(113 \mathrm{~mL})$ and $\mathbf{1 b}(1.11 \mathrm{~g}, 2.56 \mathrm{mmol})$. White crystalline solid (0.26 g, $1.25 \mathrm{mmol}, 49 \%$ ) (as well as $4 \mathbf{b}(17 \%)$ ). Mp $138-140{ }^{\circ} \mathrm{C}$ (from acetone) (lit., $\left.{ }^{72} 145-146{ }^{\circ} \mathrm{C}\right) ; R_{\mathrm{f}} 0.23$ (4:1 ether-petrol); Found: C, 80.9; $\mathrm{H}, 5.7 ; \mathrm{N}, 13.4$. Calc. for $\mathrm{C}_{14} \mathrm{H}_{12} \mathrm{~N}_{2}$ : C, 80.7; H, $5.8 ; \mathrm{N}, 13.5 \%$; IR $\nu_{\max } / \mathrm{cm}^{-1}$ (neat) 3132, 1633, 1506, 1483, $1372,1349,1268,1245,1202,1139 ; \delta_{\mathrm{H}}\left(500 \mathrm{MHz}, \mathrm{CDCl}_{3}\right.$; $\left.\mathrm{Me}_{4} \mathrm{Si}\right) 8.05$ (1H, dt ${ }_{\text {app. }}, \mathrm{H} 5 J$ 6.8, $J$ 1.2), 7.84 (2H, d, H3'/H5' $J$ 8.1), 7.77 (1H, s, H3), 7.60 (1H, dd, H8 J 9.1, $J 0.8), 7.23(2 \mathrm{H}, \mathrm{d}$, $\mathrm{H} 2$ '/H6' $J$ 8.1), 7.12 (1H, ddd, H7 J 9.1, $J$ 6.8, $J 1.3), 6.71(1 \mathrm{H}$, $\mathrm{dt}_{\text {app. }}$ H6 $J$ 6.8, $J$ 1.1), $2.38(3 \mathrm{H}, \mathrm{s}, \mathrm{Me}) ; \delta_{\mathrm{C}}\left(125 \mathrm{MHz}, \mathrm{CDCl}_{3}\right.$; $\left.\mathrm{Me}_{4} \mathrm{Si}\right)$ 145.86, 145.55, 137.72, 130.89, 129.36, 125.90, 125.45, 124.41, 117.36, 112.21, 107.69, 21.22; $\mathrm{m} / \mathrm{z}$ (ESI) $209\left([\mathrm{M}+\mathrm{H}]^{+}\right.$, 100\%). Found: $[\mathrm{M}+\mathrm{H}]^{+}$, 209.1071. $\mathrm{C}_{14} \mathrm{H}_{13} \mathrm{~N}_{2}$ requires 209.1073 .

\section{3-(4'-Methylphenyl)imidazo[1,2-a]pyridine (4b)}

Using $\mathrm{K}_{2} \mathrm{CO}_{3}$ (1.06 g, $\left.7.65 \mathrm{mmol}\right), 2$ (0.31 g, $\left.3.29 \mathrm{mmol}\right), \mathrm{PhF}$ (24 mL) and 1b (1.07 g, $2.47 \mathrm{mmol})$. White crystalline solid (0.18 g, $0.88 \mathrm{mmol}, 36 \%$ ) (as well as 3b (27\%)). Mp $84-86{ }^{\circ} \mathrm{C}$ (from DCM-ether); $R_{\mathrm{f}} 0.09$ (4:1 ether-petrol); Found: C, 80.9; $\mathrm{H}, 5.7 ; \mathrm{N}, 13.4$. Calc. for $\mathrm{C}_{14} \mathrm{H}_{12} \mathrm{~N}_{2}$ : C, 80.7; H, 5.8; N, 13.5\%; IR $\nu_{\max } / \mathrm{cm}^{-1}$ (neat) 2981, 1634, 1545, 1490, 1353, 1295, 1255, $1166,1148,1013 ; \delta_{\mathrm{H}}\left(500 \mathrm{MHz}, \mathrm{CDCl}_{3} ; \mathrm{Me}_{4} \mathrm{Si}\right) 8.30$ (1H, dt H5 $J$ 6.9, $J$ 1.2), 7.66 (1H, overlapped s, H2), 7.65 (1H, overlapped d, H8 $J$ 8.0), 7.44 (2H, d, H3'/H5' $J$ 8.0), 7.32 (2H, dd, H2'/H6' $J$ 8.0, $J$ 0.6), 7.17 (1H, ddd, H6 J 9.1, $J$ 6.9, $J$ 1.3), 6.78 (1H, td ${ }_{\text {app. }} \mathrm{H} 7 J$ 6.9, J 1.2), $2.43(3 \mathrm{H}, \mathrm{s}, \mathrm{Me}) ; \delta_{\mathrm{C}}(125 \mathrm{MHz}$, $\mathrm{CDCl}_{3} ; \mathrm{Me}_{4} \mathrm{Si}$ ) 145.99, 138.13, 132.28, 129.88, 128.00, 126.36, 125.73, 123.94, 123.34, 118.21, 112.34, 21.27. $\mathrm{m} / \mathrm{z}$ (ESI) 209 $\left([\mathrm{M}+\mathrm{H}]^{+}, 100 \%\right)$. Found: $[\mathrm{M}+\mathrm{H}]^{+}, 209.1072 . \mathrm{C}_{14} \mathrm{H}_{13} \mathrm{~N}_{2}$ requires 209.1073.

\section{2-(3'-Thienyl)imidazo[1,2-a]pyridine (3c)}

Using $\mathrm{K}_{2} \mathrm{CO}_{3}(1.06 \mathrm{~g}, 7.64 \mathrm{mmol}), 2$ (0.32 g, $\left.3.36 \mathrm{mmol}\right), \mathrm{PhF}$ $(113 \mathrm{~mL})$ and $1 \mathrm{c}(1.03 \mathrm{~g}, 2.43 \mathrm{mmol})$. White crystalline solid (0.25 g, $1.21 \mathrm{mmol}, 50 \%$ ) (as well as 4c (10\%)). Mp $163-165^{\circ} \mathrm{C}$ (from acetone); $R_{\mathrm{f}} 0.12$ (4:1 ether-petrol); Found: C, 66.1; H, 3.9; N, 13.8. Calc. for $\mathrm{C}_{11} \mathrm{H}_{8} \mathrm{~N}_{2} \mathrm{~S}$ : C, 66.0; H, 4.0; N, 14.0\%; IR $\nu_{\max } / \mathrm{cm}^{-1}$ (neat) 3124, 1632, 1508, 1476, 1338, 1306, 1272, 1242, 1144, 1090; $\delta_{\mathrm{H}}\left(500 \mathrm{MHz}, \mathrm{d}_{6}-\mathrm{DMSO} ; \mathrm{Me}_{4} \mathrm{Si}\right) 8.49(1 \mathrm{H}, \mathrm{d}$,
H5 $J$ 6.7), 8.23 (1H, s, H3), 7.89 (1H, d, H2' J 2.8), 7.61-7.55 (2H, m, H4'/H5'), 7.54 (1H, d, H8 J 9.0), 7.21 (1H, tapp. H7 J 6.6), 6.86 (1H, tapp., H6 $J$ 6.7); $\delta_{\mathrm{C}}\left(125 \mathrm{MHz}, \mathrm{d}_{6}\right.$-DMSO; $\left.\mathrm{Me}_{4} \mathrm{Si}\right)$ 144.94, 141.44, 136.38, 127.16, 127.07, 126.46, 125.15, 121.43, 116.76, 112.46, 109.31; $\mathrm{m} / \mathrm{z}$ (ESI) $201\left([\mathrm{M}+\mathrm{H}]^{+}, 100 \%\right)$. Found: $[\mathrm{M}+\mathrm{H}]^{+}, 201.0480 . \mathrm{C}_{11} \mathrm{H}_{9} \mathrm{~N}_{2} \mathrm{~S}$ requires 201.0481.

\section{3-(3'-Thienyl)imidazo[1,2-a]pyridine (4c)}

Using $\mathrm{K}_{2} \mathrm{CO}_{3}(1.07 \mathrm{~g}, 7.76 \mathrm{mmol}), 2$ (0.32 g, $\left.3.36 \mathrm{mmol}\right), \mathrm{PhF}$ $(24 \mathrm{~mL})$ and 1c $(1.05 \mathrm{~g}, 2.48 \mathrm{mmol})$. White crystalline solid (0.12 g, $0.62 \mathrm{mmol}, 25 \%$ ) (as well as 3c (33\%)). Mp $54-57{ }^{\circ} \mathrm{C}$ (from DCM); $R_{\mathrm{f}} 0.06$ (4:1 ether-petrol); IR $\nu_{\max } / \mathrm{cm}^{-1}$ (neat) 3090, 1690, 1637, 1576, 1501, 1483, 1343, 1330, 1299, 1264, $1225,1169,1154,1128,1087,1019 ; \delta_{\mathrm{H}}\left(500 \mathrm{MHz}, \mathrm{d}_{6}-\mathrm{DMSO}\right.$; $\left.\mathrm{Me}_{4} \mathrm{Si}\right) 8.61$ (1H, d, H5 J 7.0), 7.93 (1H, dd, H2' J 1.7, J 1.3), 7.85 (1H, s, H2), 7.76 (1H, dd, H5' J 5.0, J 2.1), 7.64 (1H, d, H8 J 8.5), 7.54 (1H, dd, H4' J 5.0, J 1.3), 7.29 (1H, ddd, H7 J 8.5, $J$ 6.7, J 1.7), 6.99 (1H, td $\mathrm{tapp}_{\text {. }} \mathrm{H6} J$ 6.8, $\left.J 1.1\right)$; $\delta_{\mathrm{C}}\left(125 \mathrm{MHz}, \mathrm{d}_{6}\right.$-DMSO; $\left.\mathrm{Me}_{4} \mathrm{Si}\right)$ 145.18, 132.58, 128.97, 127.20, 127.08, 124.57, 124.27, 121.18, 121.06, 117.40, 112.88; $\mathrm{m} / \mathrm{z}$ (ESI) 201 ([M + H $\left.]^{+}, 100 \%\right)$. Found: $[\mathrm{M}+\mathrm{H}]^{+}, 201.0479 . \mathrm{C}_{11} \mathrm{H}_{9} \mathrm{~N}_{2} \mathrm{~S}$ requires 201.0481 .

\section{2-(4'-Bromophenyl)imidazo[1,2-a]pyridine $(3 \mathrm{~d})^{73}$}

Using $\mathrm{K}_{2} \mathrm{CO}_{3}$ (0.96 g, $\left.6.91 \mathrm{mmol}\right), 2$ (0.28 g, $\left.2.95 \mathrm{mmol}\right)$, PhF $(102 \mathrm{~mL})$ and $1 \mathrm{~d}(1.13 \mathrm{~g}, 2.27 \mathrm{mmol})$. White crystalline solid (0.29 g, $1.47 \mathrm{mmol}, 65 \%$ ) (as well as $4 \mathrm{~d}(13 \%)$ ). Mp 196-198 ${ }^{\circ} \mathrm{C}$ (from acetone) (lit., ${ }^{73} 215-216{ }^{\circ} \mathrm{C}$ from heptane); $R_{\mathrm{f}} 0.34$ (4:1 ether-petrol); Found: C, 57.3; H, 3.2; N, 10.1. Calc. for $\mathrm{C}_{13} \mathrm{H}_{9} \mathrm{BrN}_{2}$ : C, 57.2; H, 3.3; N, 10.3\%; IR $\nu_{\max } / \mathrm{cm}^{-1}$ (neat) 2955, 2924, 1679, 1635, 1428, 1401, 1371, 1321, 1203, 1065, 1006; $\delta_{\mathrm{H}}$ (500 MHz, d $\mathrm{d}_{6}$-DMSO; $\left.\mathrm{Me}_{4} \mathrm{Si}\right) 8.51$ (1H, dt $\mathrm{app}_{\text {. }}$ H5 $J$ 6.8, $J$ 1.2), 8.42 (1H, s, H3), 7.91 (2H, d, H3'/H5' J 8.7), 7.61 (2H, d, H2'/ H6' $J$ 8.7), 7.56 (1H, dd, H8 $J$ 9.1, $J$ 1.0), 7.24 (1H, ddd, H7, $J$ 9.1, $J$ 6.8, $J 1.3$ ), 6.89 (1H, $\left.\operatorname{td}_{\text {app. }}, \mathrm{H} 6 J 6.8, J 1.0\right) ; \delta_{\mathrm{C}}(125 \mathrm{MHz}$, $\mathrm{d}_{6}$-DMSO; $\left.\mathrm{Me}_{4} \mathrm{Si}\right) 144.82,143.13,133.16,131.55,127.47$, 126.87, 125.11, 120.59, 116.61, 112.34, 109.42; $\mathrm{m} / \mathrm{z}$ (ESI) 275 $\left(\left[{ }^{81} \mathrm{Br}\right][\mathrm{M}+\mathrm{H}]^{+}, 97 \%\right), 273\left(\left[{ }^{79} \mathrm{Br}\right][\mathrm{M}+\mathrm{H}]^{+}, 100 \%\right)$. Found: $[\mathrm{M}+\mathrm{H}]^{+}$, 273.0026. $\mathrm{C}_{13} \mathrm{H}_{10} \mathrm{BrN}_{2}$ requires 273.0022.

\section{3-(4'-Bromophenyl)imidazo[1,2-a]pyridine (4d) ${ }^{69}$}

Using $\mathrm{K}_{2} \mathrm{CO}_{3}$ (1.04 g, $\left.7.55 \mathrm{mmol}\right), 2$ (0.31 g, $3.29 \mathrm{mmol}$ ), PhF $(24 \mathrm{~mL})$ and $1 \mathrm{~d}(1.23 \mathrm{~g}, 2.48 \mathrm{mmol})$. White crystalline solid (0.18 g, $0.65 \mathrm{mmol}, 26 \%$ ) (as well as 3d (29\%)). Mp 89-92 ${ }^{\circ} \mathrm{C}$ (from acetone); $R_{\mathrm{f}} 0.25$ (4:1 ether-petrol); Found: C, 57.1; $\mathrm{H}$, 3.2; N, 10.2. Calc. for $\mathrm{C}_{13} \mathrm{H}_{9} \mathrm{BrN}_{2}$ : C, $57.2 ; \mathrm{H}, 3.3 ; \mathrm{N}, 10.3 \%$; IR $\nu_{\max } / \mathrm{cm}^{-1}$ (neat) 3023, 1537, 1499, 1478, 1398, 1351, 1303, $1291,1264,1174,1151,1101,1074,1007 ; \delta_{\mathrm{H}}\left(500 \mathrm{MHz}, \mathrm{CDCl}_{3}\right.$; $\left.\mathrm{Me}_{4} \mathrm{Si}\right) 8.25\left(1 \mathrm{H}, \mathrm{dt}_{\text {app. }}, \mathrm{H} 5 J\right.$ 7.0, $J$ 1.2), 7.67 (1H, s, H2), 7.66 (1H, d, H8 J 9.1, J 1.1), 7.62 (2H, d, H2'/H6' J 8.6), $7.40(2 \mathrm{H}, \mathrm{d}$, H3'/H5' $J$ 8.6), 7.19 (1H, ddd, H7 J 9.1, $J$ 6.7, J 1.3), 6.80 (1H, $\operatorname{td}_{\text {app. }}$ H6 $J$ 6.8, $J$ 1.1); $\delta_{\mathrm{C}}\left(125 \mathrm{MHz}, \mathrm{CDCl}_{3} ; \mathrm{Me}_{4} \mathrm{Si}\right)$ 146.24, 132.68, 132.38, 129.35, 128.15, 124.49, 124.39, 123.06, 122.05, 118.29, 112.76; $m / z$ (ESI) $275\left(\left[{ }^{81} \mathrm{Br}\right][\mathrm{M}+\mathrm{H}]^{+}, 98 \%\right), 273\left(\left[{ }^{79} \mathrm{Br}\right]\right.$ $\left.[\mathrm{M}+\mathrm{H}]^{+}, 100 \%\right)$. Found: $[\mathrm{M}+\mathrm{H}]^{+}, 273.0026 . \mathrm{C}_{13} \mathrm{H}_{10} \mathrm{BrN}_{2}$ requires 273.0022 . 


\section{$1^{\prime}, 1^{\prime}$-Dibromo-2' $-\left[{ }^{13} \mathrm{C}\right]$-styrene $\left(\left[{ }^{13} \mathrm{C}\right]-19\right)^{74}$}

Triphenylphosphine $(5.04 \mathrm{~g}, 19.22 \mathrm{mmol})$ and dry carbon tetrabromide (3.10 g, $9.34 \mathrm{mmol}$ ) were dissolved in dry DCM $(30 \mathrm{~mL})$ at $0{ }^{\circ} \mathrm{C}$ under an atmosphere of nitrogen. The solution was stirred for 30 minutes before the dropwise addition of benzaldehyde $\left[{ }^{13} \mathrm{C}\right]$-carbonyl $(0.50 \mathrm{~g}, \quad 4.67 \mathrm{mmol})$ over 5 minutes. The solution was stirred at $0{ }^{\circ} \mathrm{C}$ for 1 hour before washing with an aqueous $5 \mathrm{M}$ solution of $\mathrm{CuSO}_{4}(300 \mathrm{~mL})$ followed by extraction into DCM $(3 \times 50 \mathrm{~mL})$. The organic layers were combined, dried $\left(\mathrm{MgSO}_{4}\right)$ and concentrated in vacuo. The resulting orange oily solid was dry loaded onto silica and purified by column chromatography (silica) to give the product as a pale orange clear oil which crystallized on standing $(1.21 \mathrm{~g}$, $4.60 \mathrm{mmol}, 98 \%$ ). $R_{\mathrm{f}} 0.74$ (petrol 40/60); $\delta_{\mathrm{H}}\left(300 \mathrm{MHz}, \mathrm{CD}_{2} \mathrm{Cl}_{2}\right.$; $\left.\mathrm{Me}_{4} \mathrm{Si}\right) 7.50-7.39$ (2H, m), 7.44 (1H, d, J 159.07), 7.33-7.23 (3H, $\mathrm{m}) ; \delta_{\mathrm{C}}\left(75 \mathrm{MHz}, \mathrm{CD}_{2} \mathrm{Cl}_{2} ; \mathrm{Me}_{4} \mathrm{Si}\right) 137.86$ (C1'-label). $\mathrm{m} / z$ (EI) 265 $\left(\left[{ }^{81} \mathrm{Br},{ }^{81} \mathrm{Br}\right] \mathrm{M}^{+}, 8 \%\right), 263\left(\left[{ }^{81} \mathrm{Br},{ }^{79} \mathrm{Br}\right] \mathrm{M}^{+}, 18 \%\right), 261\left(\left[{ }^{79} \mathrm{Br},{ }^{79} \mathrm{Br}\right]\right.$ $\mathrm{M}^{+}, 8 \%$ ), 184 (18), 182 (18), 103 (100). Found: $\mathrm{M}^{+}, 260.8868$. $\mathrm{C}_{7}{ }^{13} \mathrm{C}_{1} \mathrm{H}_{6}{ }^{79} \mathrm{Br}_{2}$ requires 260.8864 .

\section{Phenyl- $\alpha-\left[{ }^{13} \mathrm{C}\right]$ acetylene $\left(\left[{ }^{13} \mathrm{C}\right]-20\right)^{74}$}

$1^{\prime}, 1^{\prime}$-Dibromo-2'-[ $\left.{ }^{13} \mathrm{C}\right]$-styrene $\left(\left[{ }^{13} \mathrm{C}\right]-20\right)(1.21 \mathrm{~g}, 4.60 \mathrm{mmol})$ was dissolved in dry ether $(30 \mathrm{~mL})$ and cooled to $-78{ }^{\circ} \mathrm{C}$ under an atmosphere of nitrogen. $n$-Butyllithium $(2.17 \mathrm{M}$ in hexanes, $5.41 \mathrm{~mL}, 11.75 \mathrm{mmol}$ ) was added dropwise over 10 minutes and the solution stirred for a further 30 minutes then for 1 hour at room temperature. The reaction was quenched with water $(50 \mathrm{~mL})$, washed with water $(50 \mathrm{~mL})$ and extracted into ether $(3 \times 50 \mathrm{~mL})$. The organic layers were combined, dried $\left(\mathrm{MgSO}_{4}\right)$ and concentrated in vacuo to give the product as a pale yellow oil (0.46 g, $4.43 \mathrm{mmol}, 96 \%)$ T with sufficient purity to be used in subsequent reactions. $\delta_{\mathrm{H}}\left(300 \mathrm{MHz}, \mathrm{CDCl}_{3} ; \mathrm{Me}_{4} \mathrm{Si}\right)$ 7.54-7.49 (2H, m), 7.37-7.34 (3H, m), 3.09 (1H, d, J 49.52); $\delta_{\mathrm{C}}$ (75 MHz, $\mathrm{CDCl}_{3} ; \mathrm{Me}_{4} \mathrm{Si}$ ) 84.05 (C1'-label). m/z (EI) 103 ([M]+, $100 \%$ ). Found: $\mathrm{M}^{+}, 103.0496 . \mathrm{C}_{7}{ }^{13} \mathrm{C}_{1} \mathrm{H}_{6}$ requires 103.0498 .

\section{Phenyl(phenyl- $\beta-\left[{ }^{13} \mathrm{C}\right]$-ethynyl)iodonium trifluoroacetate $\left(\left[{ }^{13} \mathrm{C}\right]-1 \mathrm{a}\right)$}

Trifluoroacetic acid (1.01 g, $8.82 \mathrm{mmol}$ ) was added dropwise at $-30{ }^{\circ} \mathrm{C}$ to a stirred solution of phenyliodonium bis(acetate) $(1.35 \mathrm{~g}, 4.20 \mathrm{mmol})$ in dry DCM $(25 \mathrm{~mL})$ over a period of 10 minutes. After a further 30 minutes the solution was allowed to warm to room temperature and stirred for 1 hour before being re-cooled to $-30^{\circ} \mathrm{C}$ for the injection of a solution of phenyl $\left[\alpha^{-13} \mathrm{C}\right]$ acetylene, $\left[{ }^{13} \mathrm{C}\right]-20,(0.46 \mathrm{~g}, 4.43 \mathrm{mmol})$ in dry DCM $(5 \mathrm{~mL})$ over 5 minutes. The resulting mixture was then allowed to reach room temperature over 3.5 hours in darkness before concentration in vacuo (to about $5 \mathrm{~mL}$ ) followed by crystallization to give the product as a white, crystalline solid (0.63 g, $1.50 \mathrm{mmol}, 36 \%$ ). Mp 79-81 ${ }^{\circ} \mathrm{C}$ (dec.) (from DCMether-petrol) $\delta_{\mathrm{H}}\left(400 \mathrm{MHz}, \mathrm{CDCl}_{3} ; \mathrm{Me}_{4} \mathrm{Si}\right) 8.14(2 \mathrm{H}, \mathrm{d}, \mathrm{H} 2 / \mathrm{H} 6, J$ 8.7), 7.58 (1H, dt, H4, J 7.8, J 0.9), 7.49-7.39 (5H, m), $7.34(2 \mathrm{H}$, t, H3/H5 $J$ 7.3); $\delta_{\mathrm{C}}\left(100 \mathrm{MHz}, \mathrm{CDCl}_{3} ; \mathrm{Me}_{4} \mathrm{Si}\right) 162.55$ (q, (CO)

ฯ Due to volatility, solvent could not be fully removed; yield calculated from ${ }^{1} \mathrm{H}-\mathrm{NMR}$.
J 36.2), 133.55 (s, C2/C6), 132.90 (d, C3'/C5', J 2.4), 132.14 (s, C3/ C5), 131.96 (s, C4), 130.86 (d, C4', J 1.4), 128.72 (d, C2'/C6', J 5.5), 120.67 (s, C1), 120.40 (d, C1', J 86.2), 104.10 (s, C7'-label), 45.14 (d, C8', J 160.6); m/z (ESI) 306 ([M - TFA $\left.]^{+}, 100 \%\right), 294$ (14), 179 (19). Found: $[\mathrm{M}-\mathrm{TFA}]^{+}, 305.9861 . \mathrm{C}_{13}{ }^{13} \mathrm{C}_{1} \mathrm{H}_{10} \mathrm{I}$ requires 305.9855 .

\section{2-Phenyl-2/3-[ $\left.{ }^{13} \mathrm{C}\right]$-imidazo[ $[1,2-a]$ pyridine $\left(\left[{ }^{13} \mathrm{C}\right]-3 \mathrm{a}\right)$ and 3-phenyl-3- $\left[{ }^{13} \mathrm{C}\right]$-imidazo $[1,2-a]$ pyridine $\left(\left[{ }^{13} \mathrm{C}\right]-4 a\right)$}

Potassium carbonate $(0.30 \mathrm{~g}, 2.18 \mathrm{mmol})$ and 2-aminopyridine $(0.09 \mathrm{~g}, 0.97 \mathrm{mmol})$ were stirred together in dry fluorobenzene (6.3 $\mathrm{mL})$ for 45 minutes under an atmosphere of nitrogen before the addition of phenyl(phenyl $\left[\beta^{-13} \mathrm{C}\right]$ ethynyl)iodonium trifluoroacetate $(0.30 \mathrm{~g}, 0.70 \mathrm{mmol})$ by powder funnel. The solution was then stirred in darkness, at room temperature, overnight before being washed with water $(150 \mathrm{~mL})$ and extracted into DCM $(4 \times 30 \mathrm{~mL})$. The organic fractions were combined, dried $\left(\mathrm{NaSO}_{4}\right)$, filtered and concentrated in vacuo to a brown oil. The crude product was purified by column chromatography (Grace Resolve $\mathrm{TM}^{\mathrm{TM}} 80 \mathrm{~g}, 150 \mathrm{~mL}$ silica cartridge; 1:0 hexane-ether for $5 \mathrm{~min}$ then to $3: 7$ over $120 \mathrm{~min}$ and holding at this solvent mixture until elution was complete), loading the sample in DCM, to give the products as a white, crystalline solids; 2-Phenyl-2/3-[ $\left.{ }^{13} \mathrm{C}\right]$-imidazo[1,2-a]pyridine $\left(\left[{ }^{13} \mathrm{C}\right]-3 \mathrm{a}\right)(0.03 \mathrm{~g}, 0.14 \mathrm{mmol}, 20 \%)$ and 3-Phenyl-3-[ $\left[{ }^{13} \mathrm{C}\right]-$ imidazo[1,2-a]pyridine ([ $\left.\left.{ }^{\mathbf{1 3}} \mathrm{C}\right]-4 a\right)(0.04 \mathrm{~g}, 0.21 \mathrm{mmol}, 30 \%)$.

\section{2-Phenyl-2/3-[ $\left[{ }^{13} \mathrm{C}\right]$-imidazo[1,2-a]pyridine $\left(\left[{ }^{13} \mathrm{C}\right]-3 \mathrm{a}\right)$}

$R_{\mathrm{f}} 0.55$ (4:1 ether-petrol); $\delta_{\mathrm{H}}\left(400 \mathrm{MHz}, \mathrm{CDCl}_{3} ; \mathrm{Me}_{4} \mathrm{Si}\right) 8.09$ (1H, dt, H5 $J$ 6.8, J 1.2), 7.97-7.93 (2H, m, H2'/H6'), 7.84 (0.82H, dd, H3 J 8.4, $J$ 0.8), $7.84(0.18 \mathrm{H}, \mathrm{d}, \mathrm{H} 3 J$ 190.7) 7.63 (1H, d, H8 J 9.2), 7.43 (2H, tapp. $\mathrm{H}^{\prime} / \mathrm{H}^{\prime} J$ J 8.0), 7.32 (1H, t, H4' $J$ 7.6), 7.15 (1H, ddd, $\mathrm{H} 7 J$ 9.2, $J$ 6.6, J 1.2), 6.75 (1H, dt $J$ 6.6, $J$ 0.8); $\delta_{\mathrm{C}}\left(100 \mathrm{MHz}, \mathrm{CDCl}_{3} ; \mathrm{Me}_{4} \mathrm{Si}\right) 145.82$ (C2-label), 145.47 (d, C9 J 4.5), 133.76 (d, C1' J 67.9), 133.76 (s, C1'), 128.82 (d, C3'/C5' J 4.4), 128.82 (s, C3'/C5'), 128.08 (s, C4'), 126.14 (d, C2'/C6' J 2.5), 125.66 (d, C5 J 7.9), 125.66 (s, C5), 124.79 (C7), 117.62 (d, C8 J 6.1), 117.62 (s, C8) 112.55 (C6), 108.21 (C3-label); m/z (CI) 196 ([M + H $\left.]^{+}, 100 \%\right), 184$ (12). Found: $[\mathrm{M}+\mathrm{H}]^{+}$, 196.0947. $\mathrm{C}_{12}{ }^{13} \mathrm{C}_{1} \mathrm{H}_{11} \mathrm{~N}_{2}$ requires 196.0950.

\section{3-Phenyl-3- $\left[{ }^{13} \mathrm{C}\right]$-imidazo $[1,2-a]$ pyridine $\left(\left[{ }^{13} \mathrm{C}\right]-4 \mathrm{a}\right)$}

$R_{\mathrm{f}} 0.13$ (4:1 ether-petrol); $\delta_{\mathrm{H}}\left(400 \mathrm{MHz}, \mathrm{CDCl}_{3} ; \mathrm{Me}_{4} \mathrm{Si}\right) 8.31$ (1H, dd, H5 J 6.8, J 1.2), 7.68 (1H, od, H2 J 12.4), 7.65 (1H, od, H8 $J$ 8.8), 7.55-7.52 (2H, m, H2'/H6'), 7.49 (2H, tapp. H3'/H5' $J$ 8.0), 7.39 (1H, tt, H4' J 7.2, J 1.2), 7.17 (1H, ddd, H7 J 9.2, J 6.4, $J$ 1.2), $6.86\left(1 \mathrm{H}, \mathrm{t}_{\text {app. }}, \mathrm{H} 6 J 6.8\right) ; \delta_{\mathrm{C}}\left(100 \mathrm{MHz}, \mathrm{CDCl}_{3} ; \mathrm{Me}_{4} \mathrm{Si}\right)$ 146.21 (d, C9 J 9.1), 132.57 (d, C2 J 69.0), 132.52 (s, C9), 129.40 (d, C1' J 67.4), 129.33 (d, C3'/C5' J 4.2), 129.33 (s, C3 ${ }^{\prime} / \mathrm{C5}^{\prime}$ ), 128.27 (s, C4'), 128.11 (d, C2'/C6' J 2.7), 125.84 (C3-label), 124.34 (s, C7), 123.43 (s, C5), 118.09 (C8), 113.30 (C6); m/z (CI) $196\left([\mathrm{M}+\mathrm{H}]^{+}, 100 \%\right)$. Found: $[\mathrm{M}+\mathrm{H}]^{+}, 196.0945$. $\mathrm{C}_{12}{ }^{13} \mathrm{C}_{1} \mathrm{H}_{11} \mathrm{~N}_{2}$ requires 196.0950.

$\mathrm{X}$-ray crystal structures are available for compounds 3a, $3 \mathbf{a} \cdot \mathrm{HCl} \cdot \mathrm{H}_{2} \mathrm{O}, \mathbf{4 a} \cdot \mathrm{HCl} \cdot 2 \mathrm{H}_{2} \mathrm{O}$ and 7 (see ESI $\dagger$ ). CCDC 907271-907274 contain the supplementary crystallographic data for this paper. 
We thank sanofi-aventis, Newcastle University and the EPSRC (equipment grant EP/F03637X/1) for funding. We also thank Prof. W. McFarlane and Dr C. Wills for contributions in the field of NMR and the EPSRC National Mass Spectrometry Service Centre, Swansea, UK.

\section{Notes and references}

1 S. A. Galton and F. M. Beringer, J. Org. Chem., 1965, 30, 1930-1934.

2 T. Wirth, M. Ochiai, A. Varvoglis, V. V. Zhdankin, G. F. Koser, H. Tohma and Y. Kita, Hypervalent Iodine Chemistry, Springer, London, 2003.

3 P. J. Stang, Angew. Chem., Int. Ed. Engl., 1992, 31, 274-285.

4 T. Wirth, Angew. Chem., Int. Ed., 2005, 44, 3656-3665.

5 P. J. Stang and V. V. Zhdankin, Chem. Rev., 1996, 96, 1123-1178.

6 V. V. Zhdankin and P. J. Stang, Chem. Rev., 2002, 102, 2523-2584.

7 P. J. Stang, J. Org. Chem., 2003, 68, 2997-3008.

8 T. Kitamura and Y. Fujiwara, Org. Prep. Proced. Int., 1997, 29, 409-458.

9 T. Wirth and U. H. Hirt, Synthesis, 1999, 1271-1287.

10 V. V. Zhdankin and P. J. Stang, Tetrahedron, 1998, 54, 10927-10966.

11 T. Kitamura, Y. Mansei and Y. Fujiwara, J. Organomet. Chem., 2002, 646, 196-199.

12 A. Varvoglis, E. Kotali and A. Bozopoulos, J. Chem. Soc., Perkin Trans. 1, 1989, 827-832.

13 P. J. Stang and P. Murch, Tetrahedron Lett., 1997, 38, 8793-8794.

14 G. Maas, M. Regitz, U. Moll, R. Rahm, F. Krebs, R. Hector, P. J. Stang, C. M. Crittell and B. L. Williamson, Tetrahedron, 1992, 48, 3527-3540.

15 P. J. Stang, P. Murch and A. M. Arif, J. Org. Chem., 1997, 62, 5959-5965.

16 P. J. Stang, B. L. Williamson and A. M. Arif, J. Am. Chem. Soc., 1993, 115, 2590-2597.

17 M. Shimizu, Y. Takeda and T. Hiyama, Chem. Lett., 2008, 1304-1305.

18 P. J. Stang, B. L. Williamson and R. R. Tykwinski, J. Am. Chem. Soc., 1994, 116, 93-98.

19 M. Ochiai, M. Kunishima, S. Tani and Y. Nagao, J. Am. Chem. Soc., 1991, 113, 3135-3142.

20 K. S. Feldman, M. M. Bruendl and K. Schildknegt, J. Org. Chem., 1995, 60, 7722-7723.

21 I. F. D. Hyatt and M. P. Croatt, Angew. Chem., Int. Ed., 2012, 51, 7511-7514.

22 K. Banert, R. Arnold, M. Hagedorn, P. Thoss and A. A. Auer, Angew. Chem., Int. Ed., 2012, 51, 7515-7518.

23 P. J. Stang, B. W. Surber, Z. C. Chen, K. A. Roberts and A. G. Anderson, J. Am. Chem. Soc., 1987, 109, 228-235.

24 G. F. Koser, L. Rebrovic and R. H. Wettach, J. Org. Chem., 1981, 46, 4324-4326.

25 T. Kitamura and P. J. Stang, J. Org. Chem., 1988, 53, 4105-4106.
26 P. J. Stang and V. V. Zhdankin, J. Am. Chem. Soc., 1990, 112, 6437-6438.

27 M. D. Bachi, N. Barner, C. M. Crittell, P. J. Stang and B. L. Williamson, J. Org. Chem., 1991, 56, 3912-3915.

28 V. V. Zhdankin, R. Tykwinski, R. Caple, B. Berglund, A. S. Kozmin and N. S. Zefirov, Tetrahedron Lett., 1988, 29, 3717-3720.

29 V. V. Zhdankin, R. Tykwinski, B. Berglund, M. Mullikin, R. Caple, N. S. Zefirov and A. S. Kozmin, J. Org. Chem., 1989, 54, 2609-2612.

30 Y. Ishiwata and H. Togo, Synlett, 2008, 2637-2641.

31 V. V. Zhdankin, P. J. Persichini, R. Cui and Y. Jin, Synlett, 2000, 719-721.

32 V. V. Zhdankin, C. J. Kuehl, A. P. Krasutsky, J. T. Bolz and A. J. Simonsen, J. Org. Chem., 1996, 61, 6547-6551.

33 G. F. Koser, G. P. Sun, C. W. Porter and W. J. Youngs, J. Org. Chem., 1993, 58, 7310-7312.

34 M. Ochiai, Y. Masaki and M. Shiro, J. Org. Chem., 1991, 56, 5511-5513.

35 J. P. Brand, J. Charpentier and J. Waser, Angew. Chem., Int. Ed., 2009, 48, 9346-9349.

36 S. Nicolai, S. Erard, D. F. Gonzalez and J. Waser, Org. Lett., 2010, 12, 384-387.

37 E. B. Merkushev, L. G. Karpitskaya and G. I. Novosel'tseva, Dokl. Akad. Nauk SSSR, 1979, 245, 607-610.

38 L. I. Dixon, M. A. Carroll, T. J. Gregson, G. J. Ellames, R. W. Harrington and W. Clegg, Eur. J. Org. Chem., 2013, 2334-2345.

39 L. I. Dixon, M. A. Carroll, T. J. Gregson, G. J. Ellames, R. W. Harrington and W. Clegg, Org. Synth., 2013, in press.

40 Z. Liu, Z. C. Chen and Q. G. Zheng, Synth. Commun., 2004, 34, 361-367.

41 M. Ueno, T. Nabana and H. Togo, J. Org. Chem., 2003, 68, 6424-6426.

42 Y. Y. Xie, Z. C. Chen and Q. G. Zheng, J. Chem. Res., Synop., 2003, 614-615.

43 B. B. Toure, B. S. Lane and D. Sames, Org. Lett., 2006, 8, 1979-1982.

44 C. Enguehard, J.-L. Renou, V. Collot, M. Hervet, S. Rault and A. Gueiffier, J. Org. Chem., 2000, 65, 6572-6575.

45 T. Kitamura and P. J. Stang, Tetrahedron Lett., 1988, 29, 1887-1890.

46 P. J. Stang, H. Wingert and A. M. Arif, J. Am. Chem. Soc., 1987, 109, 7235-7236.

47 M. Ochiai, M. Kunishima, K. Fuji and Y. Nagao, J. Org. Chem., 1988, 53, 6144-6145.

48 M. Yoshida and S. Hara, Org. Lett., 2003, 5, 573-574.

49 M. Yoshida, A. Komata and S. Hara, J. Fluorine Chem., 2004, 125, 527-529.

50 M. Ochiai, K. Uemura, K. Oshima, Y. Masaki, M. Kunishima and S. Tani, Tetrahedron Lett., 1991, 32, 4753-4756.

51 M. Ochiai, Y. Kitagawa, M. Toyonari, K. Uemura, K. Oshima and M. Shiro, J. Org. Chem., 1997, 62, 8001-8008.

52 S. Hara, M. Yoshida, T. Fukuhara and N. Yoneda, Chem. Commun., 1998, 965-966. 
53 M. Yoshida, A. Komata and S. Hara, Tetrahedron, 2006, 62, 8636-8645.

54 T. Guan, M. Yoshida, D. Ota, T. Fukuhara and S. Hara, J. Fluorine Chem., 2005, 126, 1185-1190.

55 M. Ochiai, Y. Nishi and M. Hirobe, Tetrahedron Lett., 2005, 46, 1863-1866.

56 T. L. Ross, J. Ermert, C. Hocke and H. H. Coenen, J. Am. Chem. Soc., 2007, 129, 8018-8025.

57 P. Wipf and S. Venkataraman, J. Org. Chem., 1996, 61, 8004-8005.

58 K. Miyamoto, Y. Nishi and M. Ochiai, Angew. Chem., Int. Ed., 2005, 44, 6896-6899.

59 M. A. Carroll, S. Martin-Santamaria, V. W. Pike, H. S. Rzepa and D. A. Widdowson, J. Chem. Soc., Perkin Trans. 2, 1999, 2707-2714.

60 T. Okuyama, S. Imamura and M. Fujita, J. Org. Chem., 2006, 71, 1609-1613.

61 M. Ochiai, M. Kida, K. Sato, T. Takino, S. Goto, N. Donkai and T. Okuyama, Tetrahedron Lett., 1999, 40, 1559-1562.

62 P. Kowalski and J. Korchowiec, J. Mol. Struct. (THEOCHEM), 1993, 288, 119-131.
63 S. Nikas, N. Rodios and A. Varvoglis, Molecules, 2000, 5, 1182-1186.

64 P. J. Stang, Chem. Eng. News, 1989, 67, 4.

65 D. B. Dess and J. C. Martin, J. Am. Chem. Soc., 1991, 113, 7277-7287.

66 H. Tohma, S. Takizawa, T. Maegawa and Y. Kita, Angew. Chem., 2000, 112, 1362-1364.

67 H. J. Lucas and E. R. Kennedy, Org. Synth. Coll. Vol., 1955, 3, 485-487.

68 J. S. Yadav, B. V. Subba Reddy, Y. Gopal Rao, M. Srinivas and A. V. Narsaiah, Tetrahedron Lett., 2007, 48, 7717-7720.

69 S. Kumar and D. P. Sahu, ARKIVOC, 2008, 88-98.

70 R. Adams and I. J. Patcher, J. Am. Chem. Soc., 1954, 76, 1845-1847.

71 Gol'dfarb and Kondakowa, Zh. Prikl. Khim., 1942, 15, 151-158.

72 E. S. Hand and W. W. Paudler, Tetrahedron, 1982, 38, 49-56.

73 V. A. Artyomov, A. M. Shestopalov and V. P. Litvinov, Synthesis, 1996, 927-929.

74 E. J. Corey and P. L. Fuchs, Tetrahedron Lett., 1972, 3769-3772. 